Rev. salud pública. 6 (2):183-198

\title{
Dos Indicadores para medir la Brecha en Salud y Economía de los Países
}

\author{
Javier H. Eslava Schmalbach ${ }^{\text {I }}$, Giancarlo Buitrago Gutierrez ${ }^{\text {II }}$ \\ ${ }^{\text {I} M e ́ d i c o . ~ M . ~ S c . ~ E p i d e m i o l o g i ́ a ~ C l i ́ n i c a, ~ M . ~ S c . ~ D i r e c c i o ́ n ~ U n i v e r s i t a r i a . ~}$ \\ Instituto de Investigaciones Clínicas. Facultad de Medicina, Universidad \\ Nacional de Colombia. E-mail: jheslavas@unal.edu.co \\ II Médico. Facultad de Medicina. Universidad Nacional de Colombia. E-mail: \\ mdgiancalo@hotmail.com
}

Recibido 26 Abril 2004/Enviado para Modificación 25 Mayo 2004/Aceptado 3 Junio 2004

\section{RESUMEN}

Objetivo Desarrollar dos indicadores que permitan conocer de manera gráfica y numérica la brecha que tiene un país en la salud y en la economía.

Métodos Estudio ecológico con datos de 71 países del mundo. Se seleccionaron indicadores representativos de la salud y la economía, mediante un consenso de expertos. Para simplificar las variables que componen el modelo, se realizó un análisis de factores principales (rotación varimax). Se estimaron los índices de diferencia porcentual para cada indicador, y con la suma de las áreas de cada uno de ellos, sobre el plano Cartesiano, se estimó la brecha para la economía (IBE) y la salud (IBS) de los países. Se construyó un modelo gráfico cartesiano tanto para la brecha en salud como para la brecha en economía. Se hizo un análisis de correlación de Spearman de la brecha con el Índice de Desarrollo Humano (HDI), el Índice de Pobreza (HPI) y el Coeficiente de Gini (GINI).

Resultados La brecha en salud y económica se correlacionaron negativamente con el HDI (rho $=-0.8974$ y -0.8599 , respectivamente) y positivamente con el HPI (rho $=0.8367$, rho $=0.9196$, respectivamente) y el GINI (rho=0.5065 para IBS). IBE no se correlacionó con $\mathrm{GINI}$ (rho=0.2698).

Conclusiones Los indicadores para medir la brecha en salud y en economía de los países permiten conocer de manera gráfica el índice que más afecta la salud y la economía de cada país. Adicionalmente, gracias a sus constructos, podrían ser susceptibles de detectar variaciones pequeñas en la brecha año tras año, proceso de validación que deberá darse más adelante.

Palabras Claves: Indicadores económicos, estado de salud, países en desarrollo, países desarrollados (fuente: DeCS, BIREME). 


\begin{abstract}
Two indicators for measuring the gap between health and economics in the countries
\end{abstract}

Objective Development of two indicators to assess, in a graphical and numerical way, the gap economy and health gaps within a country.

Methods Ecologic study with data from 71 countries worldwide. Representative indicators from both health and economics were selected through an expert consensus. In order to simplify the variables that compose the model, an analysis of the main factors was carried out (varimax rotation). The difference of percentage index was estimated for each indicator, and the economic (IBE) and health gaps (IBS) were calculated from the sum of the particular areas of these indexes over a Cartesian plane. A graphic Cartesian model was constructed for each of the gaps. An analysis of Spearman correlation between IBE and IBS with the Human Development Index (HDI), the Poverty Index (HPI) and the Gini coefficient (GINI) were made.

Results IBS and IBE were negatively correlated with HDI (rho $=-0,8974$ and rho $=-0,8599$, respectively) and positively with $\mathrm{HPI}$ (rho $=-0,8367$ and rho $=0,9196$, respectively) and GINI (rho=0,5065 for IBS). IBE did not correlate with GINI ( rho=0,2698).

Conclusions The IBS and IBE indexes designed to measure the gaps in health and economy allow a graphical knowledge of the index that affects mostly the health and the economy of each country. Additionally, thanks to their constructs, they could be useful to detect small year to year variations in the gap, but this should be subject to a validation process to be carried out in the future.

Key Words: Health status, economic conditions, developing countries, developed countries (source: MeSH, NLM).

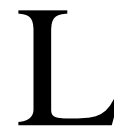

a salud y la economía de los diferentes países del mundo se han evaluado con indicadores clásicos de estructura o resultado, que permiten conocer de manera general el comportamiento en estas dos áreas, de manera no consolidada.

Los indicadores pueden ser construidos a partir de una sola variable o a partir de la reunión de mas de una variable. Estos son los llamados indicadores compuestos. Desde su introducción, por parte de Petty, en la Aritmética Política (1), o por los posteriores desarrollos de Graunt (2) o de Farr (3), los indicadores han permitido conocer el estado general de una población en un momento dado, de tal manera que facilite la toma de decisiones o la implementación de políticas. 
Sin embargo, los indicadores deben ser de fácil construcción y medición para que su aplicación pueda ser realizada por el mayor número de países, deben permitir conocer de manera rápida la situación actual del país, y deben ser susceptibles a los cambios, de tal manera que pueda graficarse su progresión en el tiempo.

Este trabajo pretende desarrollar dos indicadores compuestos para medir en un contexto global la brecha en salud (IBS) y la brecha en economía (IBE) de los países, a los que se adiciona su correspondiente expresión gráfica y matemática, que permite tener una perspectiva visual del estado del país, y de los componentes al interior de cada uno de los indicadores.

\section{METODOLOGÍA}

El trabajo se desarrolló en cuatro fases:

Fase I. Consenso de expertos para determinar los indicadores que representan la salud y la economía de los países. Para ello se realizaron dos sesiones en las que se definieron, a partir de los elementos estructurales de la salud y la economía, los indicadores que idealmente mejor las representaban. Posteriormente se cotejaron los indicadores deseados con los disponibles, de tal manera que el constructo quedó definido por consenso para los indicadores disponibles.

Los indicadores que fueron escogidos para medir la brecha de la salud y la economía de los países estuvieron caracterizados por los siguientes criterios:

- Que el consenso de expertos los considerara relevantes

- Que estuvieran reportados en más del 80 \% de los países.

- Que estuvieran disponibles en las fuentes mencionadas para el año 2000

Fase II. Estimación de los índices. Para ello se calculó cada índice a partir de cada uno de los indicadores, comparando la diferencia con el país que tenía el mejor indicador, como una proporción de la máxima diferencia existente entre todos los países. La fórmula utilizada para ello, y dependiendo de sí se trataba de una condición positiva o negativa, respectivamente, fue: 


$$
\begin{aligned}
& \text { Valor del índice }=\frac{(\text { Valor del país ideal }- \text { valor del país en cuestión })}{(\text { Valor del país ideal }- \text { valor del peor país })} \\
& \text { Ó, } \\
& \text { Valor del índice }=\frac{\text { (valor del país en cuestión }- \text { valor del país ideal })}{(\text { Valor del peor país }- \text { valor del país ideal })}
\end{aligned}
$$

Donde el país ideal, fue aquel que tenía el mejor comportamiento con respecto al indicador en cuestión. Se consideró un valor alto cuando el indicador reflejaba aspectos positivos, como por ejemplo, años de escolaridad, producto interno bruto per capita asignado a salud. En el caso de indicador negativo, se utilizó el país con un menor valor, Por ejemplo, la razón de mortalidad materna y la tasa de mortalidad en menores de 5 años.

Fase III. Análisis Factorial. Para determinar los índices que deberían quedar en el modelo final de estructura económica y en el modelo final de salud se utilizó un análisis de factores principales con la intención de disminuir ítems y dominios (mediante rotación varimax).

Fase IV. Estimación del IBE y del IBS. Se realizó una agrupación bidimensional de los índices para cada país. Se utilizó el teorema del coseno (4) para hallar el valor del lado opuesto del triángulo formado por los dos índices consecutivos, y la fórmula de Herón, para hallar el valor del área de cada triángulo a partir del valor de cada uno de sus lados así:

Teorema del coseno

$$
|a|^{2}=|b|^{2}+|c|^{2}-2|b||c| \cos (b, c)
$$

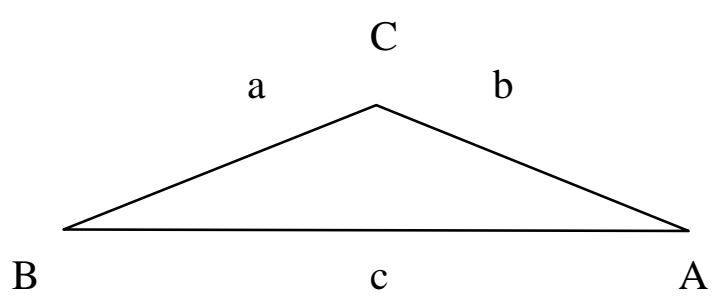

Formula de Heron (5) para hallar el área de cada uno de los triángulos: 


$$
\begin{aligned}
& \text { Eslava - Indicadores de Salud y Economía } \\
& \text { área }=\sqrt{s(s-a)(s-b)(s-c)}
\end{aligned}
$$

Donde, s es el semiperímetro, y a, b y c, corresponden a la longitud de cada uno de los lados.

$$
s=1 / 2(a+b+c)
$$

Se sumó cada una de las áreas de todos los triángulos para la estimación de la IBS y el IBE, medidos en unidades cuadráticas $\left(\mathrm{u}^{2}\right)$.

Fase V. Estudio Ecológico

Un estudio ecológico o agregado, es un estudio en el que las unidades de observación son agrupaciones de individuos. Dichas agregaciones pueden ser escuelas, fábricas, ciudades, Estados o naciones (6). Dado que se pretende hacer una análisis a partir de los datos agrupados, se propone un estudio ecológico como el diseño más adecuado para conducir este estudio.

Con los resultados de los IBS e IBE obtenidos en la fase previa, se hizo una correlación con el HDI, el HPI y el GINI. Para ello se utilizó estadística no paramétrica, dado que el comportamiento de los indicadores no fue normal (rho de Spearman).

Para la realización de este trabajo, se consideraron los 161 países incluidos en el Reporte de Desarrollo Humano 2001 de la Organización de Naciones Unidas (7). Las fuentes consultadas, fueron documentos de:

$>$ Organización Mundial de la Salud (8)

$>$ Organización Panamericana de la Salud (9)

> Banco Mundial (10)

$>$ Naciones Unidas (7)

Para el manejo de los datos se construyó una base de datos en Excel, y con ella se hicieron los cálculos matemáticos. El análisis estadístico se hizo con STATA versión 6.0.

\section{RESULTADOS}

Los indicadores seleccionados que cumplieron los criterios de inclusión y que fueron seleccionados por los expertos, se muestran en la Tabla 1. 
Índice de Brecha en Salud (IBS)

El 89,2 \% de la varianza para IBS se explica en los dos primeros factores, lo que se aprecia en la Tabla 2. Con el análisis factorial se excluyen en la construcción del IBS los indicadores Toneladas de CO2 y Consumo de Cigarrillo por adulto. Las variables quedan distribuidas en dos dominios, como se aprecia en la Tabla 3. El coeficiente de reproducibilidad Alpha de Chronbach, para IBS fue de 0.8910 .

A manera de ejemplo se muestra la gráfica del IBS para Colombia y Guinea (Figuras 1 y 2 ).

Tabla 1. Indicadores que cumplieron los criterios de inclusión

\begin{tabular}{|c|c|}
\hline \multirow{2}{*}{\multicolumn{2}{|c|}{ IBE }} \\
\hline & \\
\hline PIB per cápita para salud. & Tasa de mortalidad de niños menores de \\
\hline PIB per cápita para educación. & un año. \\
\hline Niños que trabajan entre 10 y 15 años & Tasa de mortalidad de niños menores de \\
\hline Población Urbana. & cinco años. \\
\hline Tasa de alfabetización de adultos. & Adultos viviendo con VIH/SIDA. \\
\hline Tasa bruta de matriculación primaria, & Casos de TBC. \\
\hline secundaria y terciaria combinados. & Consumo de cigarrillos por adulto. \\
\hline Población con servicios de saneamiento & Niños con bajo peso al nacer. \\
\hline adecuados. & Años de vida sana. \\
\hline Población con fuentes de aguas & Toneladas de $\mathrm{CO}_{2}$ per cápita. \\
\hline mejoradas. & Médicos por 100.000 hab. \\
\hline $\begin{array}{l}\text { Tasa de crecimiento anual de la } \\
\text { población. }\end{array}$ & $\begin{array}{l}\text { Población con acceso a medicamentos } \\
\text { esenciales. }\end{array}$ \\
\hline & $\begin{array}{l}\text { Niños de un año totalmente inmunizados } \\
\text { contra sarampión. }\end{array}$ \\
\hline
\end{tabular}

Los valores extremos del IBS estuvieron en Estados Unidos (menor IBS : $0.0024796 \mathrm{u}^{2)}$ y Guinea-Bissau (mayor IBS: $1.3005223 \mathrm{u}^{2}$ ). La mediana de la distribución estuvo en 0.1358972 .

Los países que se encuentran en el quintil inferior del IBS (con menos brecha en salud) son, de menor a mayor: Estados Unidos (0.00248 u2), Italia (0.04004 u2), España (0.010101 u2), Grecia (0.14049 u2), Grecia (0.014049 u2), Suecia (0.018654 u2), Israel (0.019988 u2), Francia (0.021462 u2), Finlandia (0.027075 u2). Los países que se encuentran en el quintil superior de IBS (mayor brecha) son de menor a mayor: Zimbawe (0.634754 u2), Côte d'lvoire (0.68949 u2), Togo (0.703565 u2), Camerún (0.71523 u2), Mauritania (0.72888 u2), India (0.766536 u2), Kenya (0.791821 u2), Guinea-Bissau (1.300522 u2). 
Tabla 2. Tabla de Valores Propios para IBS (Análisis Factorial)

\begin{tabular}{ccccc}
\hline Factor & $\begin{array}{c}\text { Valores } \\
\text { Propios }\end{array}$ & Diferencia & Proporción & Acumulado \\
\hline $\mathbf{1}$ & $\mathbf{6 . 5 2 4 5 8}$ & $\mathbf{5 . 6 3 9 1 4}$ & $\mathbf{0 . 7 8 5 6}$ & $\mathbf{0 . 7 8 5 6}$ \\
$\mathbf{2}$ & $\mathbf{0 . 8 8 5 4 4}$ & $\mathbf{0 . 2 2 2 2 6}$ & $\mathbf{0 . 1 0 6 6}$ & $\mathbf{0 . 8 9 2 2}$ \\
$\mathbf{3}$ & 0.66318 & 0.26272 & 0.0798 & 0.9720 \\
$\mathbf{4}$ & 0.40046 & 0.23852 & 0.0482 & 1.0203 \\
5 & 0.16194 & 0.05673 & 0.0195 & 1.0398 \\
6 & 0.10521 & 0.09568 & 0.0127 & 1.0524 \\
7 & 0.00953 & 0.01781 & 0.0011 & 1.0536 \\
8 & -0.00828 & 0.03218 & -0.0010 & 1.0526 \\
9 & -0.04046 & 0.05798 & -0.0049 & 1.0477 \\
10 & -0.09844 & 0.02947 & -0.0119 & 1.0359 \\
\hline
\end{tabular}

Tabla 3. IBS. Representación de ítems y dominios

\begin{tabular}{cllr}
\hline Dominio & $\begin{array}{c}\text { Carga } \\
\text { Factorial }\end{array}$ & \multicolumn{1}{c}{ Item } & Unicidad \\
\hline & 0.95255 & Mortalidad menores de cinco años & -0.00125 \\
Acceso & 0.95721 & Mortalidad menores de un año & -0.00115 \\
& 0.40612 & Niños con bajo peso al nacer & 0.46751 \\
& 0.80399 & Años de vida sana & 0.07974 \\
& 0.43673 & Médicos por 100.000 hab. & 0.34620 \\
& 0.44742 & Acceso a drogas esenciales & 0.40634 \\
& 0.62897 & Niños inmunizados contra & 0.43252 \\
\hline \multirow{2}{*}{$\begin{array}{c}\text { Transmisibl } \\
\text { es }\end{array}$} & 0.75633 & Adultos viviendo con vih/sida & 0.29479 \\
\hline
\end{tabular}

Índice de Brecha Económica (IBE)

El 95,4 \% de la varianza para IBE, se explica en los dos primeros factores, lo que se aprecia en la Tabla 4. Las variables de IBE quedan distribuidas en dos dominios como se aprecia en la Tabla 5. El coeficiente de reproducibilidad Alpha de Chronbach, para IBE fue de 0.9106.

Se muestra la figura del IBE para Colombia y Níger, durante los mismos periodos de tiempo (Figuras 3 y 4 ).

Los valores extremos de IBE estuvieron en Suiza (menor IBE: 0.0210665 $\mathrm{u}^{2}$ ) y Níger (Mayor IBE: $2.0035565 \mathrm{u}^{2}$ ). La mediana de la distribución estuvo en $0.60779 \mathrm{u}^{2}$. 
Figura 1. IBS para Colombia (IBS: $0.123 \mathrm{u}^{2}$ )

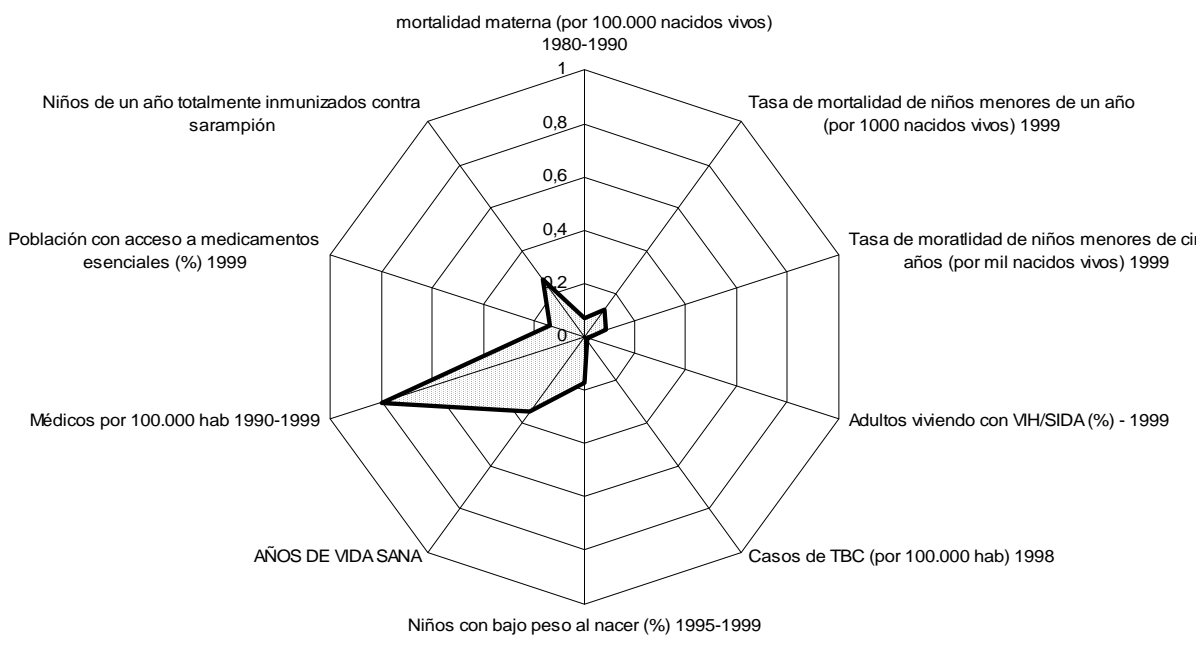

Figura 2. IBS para Guinea. (IBS:1.3005322 u²)

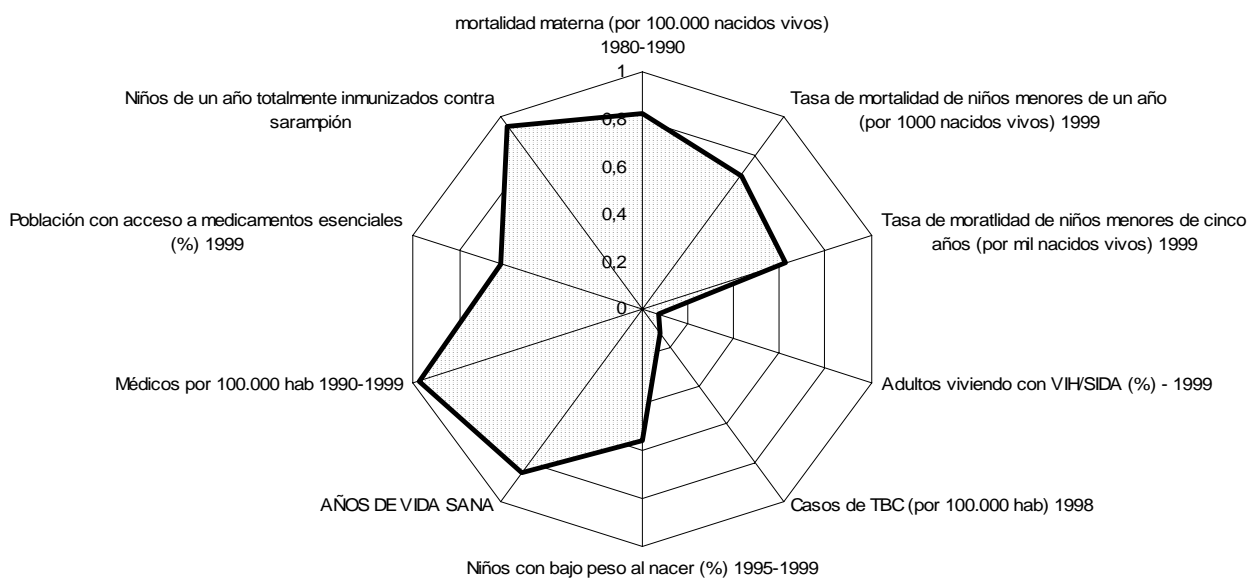


Tabla 4. Valores propios para IBE (Análisis factorial)

\begin{tabular}{ccccc}
\hline Factor & Valores Propios & Diferencia & Proporción & Acumulado \\
\hline 1 & 4.94873 & 3.80122 & 0.7751 & 0.7751 \\
2 & 1.14751 & 0.80969 & 0.1797 & 0.9548 \\
3 & 0.33782 & 0.05341 & 0.0529 & 1.0077 \\
4 & 0.28441 & 0.13248 & 0.0445 & 1.0523 \\
5 & 0.15193 & 0.11964 & 0.0238 & 1.0761 \\
6 & 0.03229 & 0.06336 & 0.0051 & 1.0811 \\
7 & -0.03107 & 0.09870 & -0.0049 & 1.0762 \\
8 & -0.12977 & 0.02717 & -0.0203 & 1.0559 \\
9 & -0.15694 & 0.04313 & -0.0246 & 1.0313 \\
10 & -0.20008 &. & -0.0313 & 1.0000 \\
\hline
\end{tabular}

Los países que se encuentran en el quintil inferior del IBE (con menos brecha en economía) son de menor a mayor: Suiza (0.0210664 u2), Suecia (0.0311019 u2), Finlandia (0.0699999 u2), Estados Unidos (0.0763656 u2), Canadá (0.0764398 u2), Austria (0.0830205 u2), Países Bajos (0.0830298 u2), Reino Unido (0.0852665 u2). Los países que se encuentran en el quintil superior de IBE (mayor brecha) son de menor a mayor: Benin (1.477812 u2), Guinea (1.4877854 u2), Madagascar (1.5060610 u2), Mali (1.5049322 u2), Camboya (1.5792153 u2), Nepal (1.6065722 u2), Chad (1.7422635 u2), Etiopía (18707574 u2), Níger (2.0035565 u2).

Tabla 5. IBE. Representación de ítem y dominios

\begin{tabular}{cclc}
\hline \multirow{2}{*}{ Dominio } & Carga factorial & \multicolumn{1}{c}{ Ítem } & Unicidad \\
\hline \multirow{3}{*}{ Económico } & 0.82051 & PIB per capita & 0.20145 \\
& 0.63353 & PIB per capita salud & 0.33734 \\
& 0.41281 & PIB per capita educación & 0.53872 \\
& 0.63234 & Matricula prim. secun. terc. & 0.47973 \\
\hline \multirow{5}{*}{ Social } & 0.90028 & Niños trabajando (10-15 años) & 0.13987 \\
& 0.67209 & Población urbana & 0.30987 \\
& 0.84294 & Tasa de alfabetización & 0.17622 \\
& 0.78802 & Servicios de saneamiento & 0.24575 \\
& 0.70961 & Servicio de aguas tratadas & 0.28732 \\
& 0.51902 & Crecimiento anual de la & 0.17249 \\
\hline
\end{tabular}


Figura 3. IBE para Colombia. (IBE: $0,36696 \mathrm{u}^{2}$ )

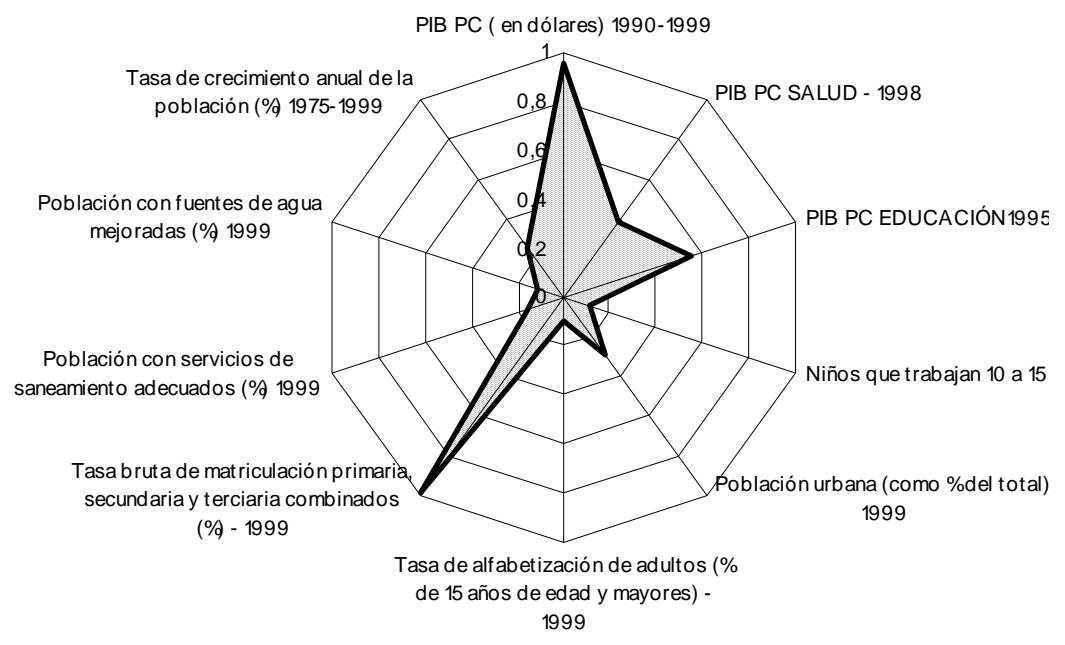

Figura 4. IBE para Niger. IBE: $2.0035565 \mathrm{u}^{2}$

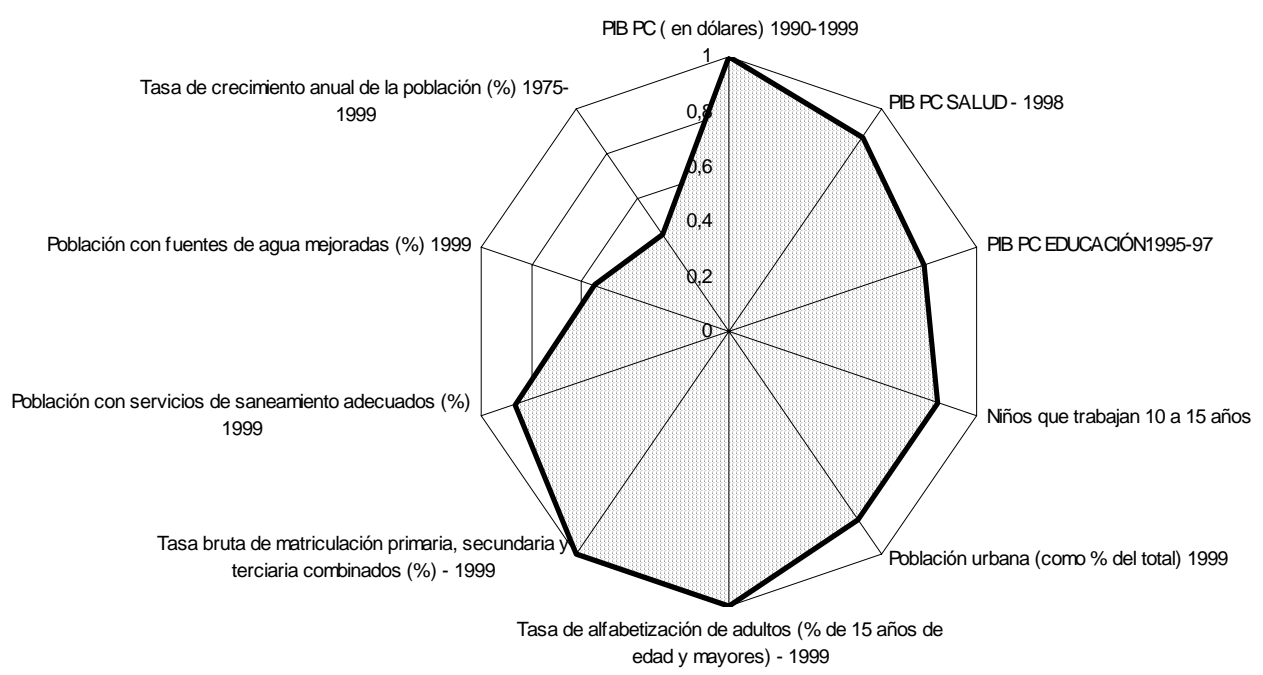


Análisis de correlación

La correlación de Spearman entre el IBS e IBE, con el HDI, el HPI y el GINI, se presentan en la Tabla 6. La brecha en salud y económica se correlacionaron de manera inversa con el HDI (rho $=-0.8974$ y -0.8599 , respectivamente), como era de esperarse por la connotación positiva y negativa de cada indicador y positivamente (por la connotación negativa de ambos indicadores) con el HPI y el GINI. No hubo correlación entre IBE y GINI (rho=0.2698).

Tabla 6. Coeficientes de correlación (rho de Spearman)

\begin{tabular}{ccccc}
\hline & IBE & HDI & HPI & GINI \\
\hline IBS & 0.74 & -0.8974 & 0.8367 & 0.5065 \\
IBE & & -0.9196 & 0.9196 & 0.2698 \\
HDI & & & -0.9540 & -0.4523 \\
HPI & & & & -0.2650 \\
\hline
\end{tabular}

\section{DISCUSIÓN}

Este trabajo permitió el desarrollo de dos indicadores, uno para medir la brecha o ventana en la economía y otro, la brecha o ventana en la salud de los países. En virtud que fueron construidos con base en un patrón de referencia, la brecha se estima a partir de la distancia que existe en el mundo para cada una de las variables que componen el indicador en el país, con respecto a la distancia que existe para esa misma variable entre el peor y el mejor país del mundo, para ese mismo año.

Con base en lo anterior se espera que el modelo que a priori es comparativo, sea dinámico en el sentido que países que no progresan en la mejoría de sus variables, presentarán en el tiempo un peor comportamiento, si la situación en el resto del mundo tiende hacia la mejoría. En dicho caso, la distancia proporcional con el patrón de referencia aumentará, aunque la variable para el país no haya presentado modificaciones sustanciales.

Adicionalmente, mejorías sustanciales de una variable en un país, que se sitúan a la par con mejoras sustanciales en la situación de los países del resto del mundo, no permitirán visualizar una disminución en la brecha para la variable particular. Sin embargo, como se trata de un indicador compuesto, la 
brecha global aumentará cuando todas o varias de las variables que componen el modelo se queden estáticas ante una mejoría de ellas en el mundo, o cuando una o varías de las que componen el modelo, presenten un empeoramiento global relativo, al resto de la situación del mundo.

La representación gráfica de las distancias de cada una de las variables que construyen con respecto al patrón de referencia, permiten apreciar visualmente el aspecto más relevante en salud o economía que está aumentando la brecha en cada país contra el país usado para construir el patrón de referencia. De tal manera que podría decirse, que todos los países fueron comparados contra un país ideal, creado a partir de las variables con mejor comportamiento en el contexto mundial, de tal manera que el IBS y el IBE para dicho país ideal será de $0 \mathrm{u}^{2}$.

El IBE quedó entonces distribuido en dos dominios, uno principalmente monetario y el otro social. Es llamativo que la educación quedó representada por una variable diferente, en cada uno de los dominios.

El IBS quedó distribuido también en dos dominios, uno que agrupa los indicadores de mortalidad y acceso a los servicios, y el otro que agrupa las enfermedades transmisibles.

En el caso de la tasa de crecimiento anual de la población, fue difícil establecer cuál era la mejor tasa de crecimiento, considerando que los países con tasas negativas tienden a invertir su pirámide poblacional y a favorecer una población de adultos mayores no productivos que podrían perjudicar la economía del país. En el caso contrario, tasas de crecimiento anual altas, podrían afectar la distribución de los recursos entre la población, en caso de recursos escasos, lo que a la postre también resultaría deletéreo. En dicho caso, luego de consultar al consenso, se determinó que una tasa positiva de crecimiento de 0.4 , era ideal.

Nightingale, en 1858 (11), había diseñado una presentación gráfica bidimensional similar (Bat's wing diagram, o diagrama de alas de murciélago), de las causas de mortalidad en el Este, en donde la longitud del brazo radial para cada mes era proporcional a la tasa de muerte.

En 1997, Greiner y Rose enviaron para presentación un trabajo al "21st Annual German Conference on AI'97-Knowledge Management”, en el que se desarrolló el concepto de "Visual Health Profile", que tiene similaridades con la presentación gráfica de IBS e IBE. Dicha presentación, aunque fue 
aceptada, no fue presentada en el evento (12). Tampoco desarrolló un modelo comparativo ni matemático, entre los diferentes países.

El Índice de Desarrollo Humano (HDI) fue desarrollado por el Programa de Desarrollo de las Naciones Unidas, y es una medida resumen de tres indicadores clásicos: la deprivación en la educación, la deprivación en la expectativa de vida y la deprivación en el Producto Interno Bruto (7). El HDI se ha constituido con un patrón de referencia para comparar a los diferentes países del mundo(13). Sin embargo, se espera que cambie de manera lenta en el tiempo, por las variables con las que fue construido, lo que lo hace entonces, poco sensible a los cambios anuales que puedan presentarse en la estructura económica o en los desenlaces de salud en un país determinado. Adicionalmente, es un número construido de manera unidimensional, como el complemento del promedio de las tres deprivaciones, lo que imposibilita conocer cual afecta en mayor o menor grado el resultado del mismo. Igual situación puede comentarse con respecto al Índice de Pobreza (HPI).

Existen otros indicadores que dan cuenta de aspectos del desarrollo puntual económico o social de las poblaciones, y que son los que se utilizan y publican de manera aislada o comparada por organismos como la Organización Mundial y la Organización Panamericana de la Salud, el Banco Mundial, entre otros $(9,14)$.

Un indicador será más útil en la medida que permite entender fácilmente que pasa con la situación del país, y para ello la representación gráfica es muy útil, dado que permite visualizar las condiciones particulares de cada una de la variables que producen el resultado. También será más útil cuando puede ser medido por la mayoría de países, y cuando es sensible al cambio año tras año. Además es fundamental para su validez, que en realidad mida lo que dice medir.

Aunque este trabajo, por tratarse de un estudio ecológico, se soporta en la veracidad de la información obtenida a través de las fuentes consultadas, éstas mismas fuentes son las que utilizan los organismos internacionales para evaluar la gestión y en las que muchas veces se fundamenta la toma de decisiones, e inversión de recursos en el escenario internacional.

Este modelo de indicador gráfico y matemático de la brecha de salud y economía, llega a posicionar a cada país en el contexto mundial, de tal suerte que se puede conocer la magnitud comparativa de su brecha, y el indicador que tiene el mejor y peor comportamiento en salud y en economía dentro de la brecha. Aunque este trabajo no llegó hasta ese nivel de desarrollo del indi- 
cador, también es factible extrapolar la información de las unidades cuadráticas obtenidas, a un índice con valores entre 0 y 1 , de tal manera que permita hacer una comparación mental más fácil de los datos numéricos.

Con este modelo se rescatan elementos matemáticos útiles para la diagramación de otras brechas o ventanas, en otros escenarios, que puedan facilitar la toma de decisiones en los escenarios respectivos.

En lo que respecta a su validez, la comparación con los otros indicadores disponibles para medir la economía o la salud de una manera directa o indirecta, mostraron una alta correlación de IBE e IBS con el HDI y el HPI. El IBS presentó una alta correlación con el GINI, cosa que no ocurrió con el IBE, ni con el HPI. El HDI tiene también una relación alta negativa con el GINI. Estas relaciones se explican de manera lógica por la forma como están construidos los indicadores para comparación. De tal manera que el HDI mide de manera positiva, el nivel de desarrollo que tienen los países, mientras que el IBS y el IBE miden la brecha, o en otras palabras, lo que les sobra de negativo a cada uno de las variables que componen el modelo de brecha en salud o de brecha en economía. Es por eso que la correlación es negativa entre IBS e IBE con HDI, y positiva con HPI.

Comparativamente, tanto el IBS como el IBE ofrecen la posibilidad de ver gráficamente el tamaño de la brecha y dentro de sí mismos, conocer cuál es la variable con el peor o mejor comportamiento. De ahí su utilidad, por encima de los indicadores existentes.

Considerando las tendencias globalizadoras actuales, el posicionamiento del país dentro de un contexto mundial, es útil como referente del tamaño de su lejanía a la verdadera condición de desarrollo.

Es objetivo de otras fases de investigación de este mismo trabajo comparar la sensibilidad al cambio, dentro de los países de estos dos indicadores, de tal manera que pueda vislumbrarse el aumento o la disminución de la brecha como resultado de los condicionantes políticos, económicos y sociales en el que están inmersos los países, aunque se presume, por las variables incluidas en su construcción, que es susceptible de mostrar variaciones año tras año.

Finalmente se puede afirmar que IBS e IBE son dos indicadores válidos que permiten conocer de manera gráfica y matemática la brecha en salud y en economía de los países en el contexto mundial 
Agradecimientos. A los profesores Hernando Gaitán, Ricardo Sánchez, Jorge Díaz, Rodrigo Pardo y a la Economista Ana Gómez, por sus invaluables aportes durante la Fase de Consenso de Expertos. A Mario Moreno quien hizo parte de la línea de profundización en Economía en Salud. Este estudio fue apoyado por el programa Semilleros de Investigación de la Universidad Nacional de Colombia.

\section{REFERENCIAS}

1. Petty W. Political arithmetick. London, Printed for Robert Clavel at the Peacock, and Hen. Mortlock at the Phoenix in St. Paul's Church-yard, Londres, 1690. Texto electrónico preparado por Rod Hay y publicado por el Archivo de Pensamiento Económico de la Universidad de McMaster, Abril 1, 1998. [Internet] Disponible en http://socserv2.socsci.mcmaster.ca/ econ/ugcm/ 3ll3/petty/ poliarith.html. Consultado en abril de 2004.

2. Graunt J. Natural and political observations. Mentioned in a following Index, and made upon the Bills of Mortality. Printed by Tho: Roycroft, for John Martin, James Allestry, and Tho: Dicas, at the Sign of the Bell in St. Paul's Church-yard, 1662. London. Texto electrónico preparado por Ed Stephan 25 Jan 96. [Internet] Disponible en http://www.ac.wwu.edu/ stephan/ Graunt/0.html. Consultado en Abril de 2004

3. Farr W. (1838) On Prognosis. British Medical Almanack; Supplement 199-216. Typed and edited by Gerry Bernard Hill. Series: History of epidemiology. [Internet] Disponible en http://www.epidemiology.ch/ history/papers/ SPM48(4)219-24Farr_Part1.pdf y en http://www.Epidemio logy.ch/history/ papers/SPM48(5)279-84Farr_Part2.pdf. Consultado en Abril de 2004.

4. Euclid. Euclid's Elements. Libro II, Proposición 12. Edición electrónica por Joyce, D. Clark University. [Internet] Disponible en http://aleph0.clarku. edu/ djoyce/java/elements/bookII/propII12.html; 1996 Consultado en Marzo de 2004.

5. School of Mathematics and Statistics. University of St Andrews, Scotland. Heron of Alexandria. JOC/EFR; 1999. [Internet] Disponible en http://wwwhistory.mcs.st-and.ac.uk/history/Mathematicians/Heron.html Consultado en Diciembre de 2003.

6. Rothman K, Greenland S. Types of epidemiologic studies. In: Rothman K, Greenland S. Modern Epidemiology. Philadelphia: Lippincott-Raven Publishers. 1998. p.77.

7. Human Development Report. United Nations Development Program. New York. Oxford University Press; 2001.

8. World Health Organization. [Internet]. Disponible en www.who.int/en/ Consultado en Marzo de 2004.

9. Panamerican Health Organization [Internet]. Disponible en www.paho.org Consultado en Febrero de 2004.

10. Informe Sobre el Desarrollo Mundial 2000/2001. Banco Internacional de Reconstrucción y Fomento. Banco Mundial. Washington, D.C; 2001. 
11. Nightingale F. Notes on Matters affecting the Health Efficiency, and Hospital Administration of the British Army, founded chiefly on the experience of the late war. Harrison and sons, London; 1858. In: Smith D. Measurement tips from Florence Nightingale. Have we learned anything new in the last 150 years. The MITRE Corporation. USA; 2000 [Internet]. Disponible en www.mitre.org/work/tech-papers Consultado en marzo de 2004.

12. Greiner C, Rose T. The Health Profile. In: Knowledge Management in Global Health Research Planning. Aceptado para presentación en el Workshop "Knowledge-Based Systems for Knowledge Management in Enterprises" 21st Annual German Conference on AI '97. (KI-Jahrestagung '97). September 9th - 12th. Freiburg, Baden-Württemberg, Germany; 1997 [Internet]. Disponible en www.dfki.uni-kl.de/ aabecker/Freiburg/Final/ Greiner/ greiner.html

13. Human Development Report. Technical Notes. United Nations Development Program. New York. Oxford University Press; 1997. p.117-128

14. World Health Organization. Country Indicators. Selected Health Indicators for Colombia [Internet]. Disponible en http://www3.who.int/whosis/country/ indicators.cfm?country=col; 2002 Consultada en Diciembre de 2004. 